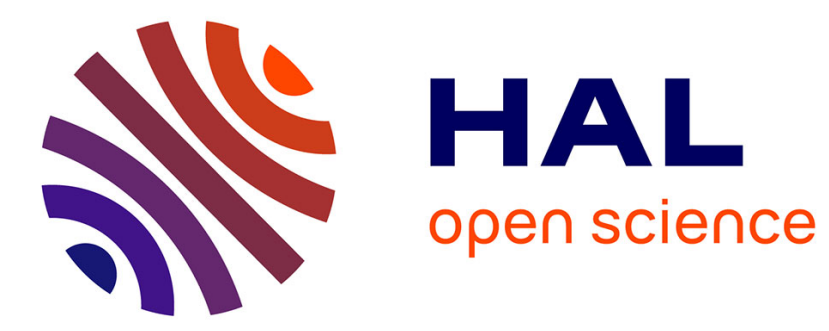

\title{
Ciprofloxacin Prevents Myelination Delay in Neonatal Rats Subjected to E. coli Sepsis
}

Gauthier Loron, Paul Olivier, Hélène See, Nolwenn Le Saché, Leslie Angulo, Valérie Biran, Nadège Brunel, Bernadette Besson-Lescure, Marie-Dominique Kitzis, Julien Pansiot, et al.

\section{To cite this version:}

Gauthier Loron, Paul Olivier, Hélène See, Nolwenn Le Saché, Leslie Angulo, et al.. Ciprofloxacin Prevents Myelination Delay in Neonatal Rats Subjected to E. coli Sepsis. Annals of Neurology, 2010, 10.1002/ana.22190 . hal-02470193

\section{HAL Id: hal-02470193 https://hal.sorbonne-universite.fr/hal-02470193}

Submitted on 20 Feb 2020

HAL is a multi-disciplinary open access archive for the deposit and dissemination of scientific research documents, whether they are published or not. The documents may come from teaching and research institutions in France or abroad, or from public or private research centers.
L'archive ouverte pluridisciplinaire HAL, est destinée au dépôt et à la diffusion de documents scientifiques de niveau recherche, publiés ou non, émanant des établissements d'enseignement et de recherche français ou étrangers, des laboratoires publics ou privés. 


\title{
Ciprofloxacin Prevents Myelination Delay in Neonatal Rats Subjected to E. coli Sepsis
}

\author{
Gauthier Loron, MD, ${ }^{1}$ Paul Olivier, PhD, ${ }^{1}$ Hélène See, MD, ${ }^{1,2}$ Nolwenn Le Saché, MD, ${ }^{1,2}$ \\ Leslie Angulo, ${ }^{1,2}$ Valérie Biran, MD, PhD, ${ }^{1,7,8}$ Nadège Brunelle, PhD, ${ }^{3}$ \\ Bernadette Besson-Lescure, PhD, ${ }^{3}$ Marie-Dominique Kitzis, PhD, ${ }^{4}$ Julien Pansiot, MS, ${ }^{1}$ \\ Edouard Bingen, MD, ${ }^{2,5}$ Pierre Gressens, MD, PhD, ${ }^{1,6,7}$ Stéphane Bonacorsi, MD, PhD, ${ }^{2,5}$ \\ and Olivier Baud, MD, PhD ${ }^{1,7,8}$
}

Objective: Perinatal infections and the systemic inflammatory response to them are critical contributors to white matter disease (WMD) in the developing brain despite the use of highly active antibiotics. Fluoroquinolones including ciprofloxacin (CIP) have intrinsic anti-inflammatory effects. We hypothesized that CIP, in addition to its antibacterial activity, could exert a neuroprotective effect by modulating white matter inflammation in response to sepsis.

Methods: We adapted an Escherichia coli sepsis model to 5-day-old rat pups (P5), to induce white matter inflammation without bacterial meningitis. We then compared the ability of CIP to modulate inflammatory-induced brain damage compared with cefotaxime (CTX) (treatment of reference).

Results: Compared with CTX, CIP was associated with reduced microglial activation and inducible nitric oxide synthase (iNOS) expression in the developing white matter in rat pups subjected to E. coli sepsis. In addition to reducing microglial activation, CIP was able to prevent myelination delay induced by $E$. coli sepsis and to promote oligodendroglial survival and maturation. We found that $E$. coli sepsis altered the transcription of the guidance molecules semaphorin 3A and 3F; CIP treatment was capable of reducing semaphorin 3A and 3F transcription levels to those seen in uninfected controls. Finally, in a noninfectious white matter inflammation model, CIP was associated with significantly reduced microglial activation and prevented WMD when compared to CTX.

Interpretation: These data strongly suggest that CIP exerts a beneficial effect in a model of E. coli sepsis-induced WMD in rat pups that is independent of its antibacterial activity but likely related to iNOS expression modulation.

ANN NEUROL 2011;69:341-351

W hite matter disease (WMD) is the major pathology underlying the cerebral palsy and cognitive impairment observed in very preterm neonates who survive the intensive care unit. In the most recent epidemiological reports, the incidence of WMD in infants with a gestational age of less than 32 weeks is as high as $20 \%{ }^{1,2}$ Several perinatal factors, including maternal or neonatal infection, hypoxia-ischemia, endocrine imbalances, and

genetic factors have been implicated in the pathophysiology of brain lesions associated with WMD. ${ }^{3}$

Perinatal infections resulting in an excess of cytokines and other proinflammatory agents are one of the most striking contributors to WMD in the developing brain. ${ }^{4}$ Infants with sepsis have recently been shown to have a very high incidence (80\%) of white matter abnormalities. ${ }^{5,6}$ Escherichia coli is one of the main pathogens

View this article online at wileyonlinelibrary.com. DOI: 10.1002/ana.22190

Received Feb 23, 2010, and in revised form Jul 17, 2010. Accepted for publication Jul 30, 2010.

Address correspondence to Dr Baud, INSERM, AVENIR-U676, Hôpital Robert Debré, 48 Blvd Sérurier, F-75019 Paris, France.

E-mail: olivier.baud@rdb.aphp.fr

From the ${ }^{1}$ Institut National de la Santé et de la Recherche Médicale (National Institute of Health and Medical Research) AVENIR R05230HS, ${ }^{5}$ Department of Microbiology, ${ }^{6}$ INSERM UMR 676, Université Paris Diderot, ${ }^{8} \mathrm{NICU}$, Université Paris Diderot, Hôpital Robert Debré, Assistance Publique-Hôpitaux de Paris (APHP), Paris, France; ${ }^{2}$ Equipe d'accueil EA 3105, Université Paris Diderot, Paris, France; ${ }^{3}$ INSERM IFR 65 IRSSA, Hôpital Saint Antoine, APHP, Paris, France;

${ }^{4}$ Fondation Hôpital Saint-Joseph, Paris, France; ${ }^{7}$ PremUP, Paris, France. 
causing early-onset infections in preterm neonates, accounting for up to $40 \%$ of the cases of bacteremia among very low birth weight preterm infants $(<1,500 \mathrm{~g}) .^{7}$ During the last decade, the incidence of early-onset $E$. coli infections has increased to $0.5 \%$ to $1 \%$ in this high-risk population. ${ }^{7,8}$

Fluoroquinolones are antibiotics with bactericidal activity that inhibit DNA gyrase, an enzyme essential for bacterial DNA synthesis. These antibiotics, although not approved for use in neonates, have been previously used with success in multidrug-resistant or complicated Gram-negative infections, with no major adverse effects. ${ }^{9,10}$ Indeed, this class of drugs has several advantages compared to beta-lactam antibiotics, the antibacterial agents of reference in neonatal infections, such as bactericidal activity on stationary-phase bacteria and good tissue diffusion, especially in the central nervous system (CNS). ${ }^{11}$ However, ciprofloxacin (CIP) has only a moderate efficacy against Gram-positive bacteria, especially Group B Streptococcus and cannot replace cefotaxime (CTX) in a context of empiric antibiotic therapy.

In addition to their antibacterial activity, fluoroquinolones possess intrinsic anti-inflammatory effects, decreasing the expression of pro-inflammatory cytokines and inducible nitric oxide synthase (iNOS). ${ }^{12-14}$ In very preterm infants at high risk of developing brain lesions, the use of such antibiotics, combining antibacterial activity and anti-inflammatory properties, would constitute an original approach, since perinatal inflammation is considered one of the strongest predictors of WMD.

Here, we hypothesized that the intrinsic anti-inflammatory properties of the fluoroquinolone CIP may have neuroprotective effects in the developing brain subjected to E. coli sepsis. Using an animal model characterized by perinatal $E$. coli sepsis without meningitis, we demonstrate that systemic sepsis is associated with inflammation and transient myelination defects in the immature white matter. Compared to CTX as the treatment of reference, CIP is able to prevent WMD through the modulation of white matter inflammation in response to $E$. coli sepsis.

\section{Subjects and Methods}

\section{Bacterial Strains}

E. coli strain C5 was kindly provided by Robert Bortolussi (Dalhousie University, Halifax, Canada), and is representative of the worldwide highly virulent clonal group O18:K1:H7 that causes neonatal bacteremia and meningitis. ${ }^{15} \mathrm{C} 5$ is sensitive to all antibiotics, and minimal inhibitory concentrations for CTX and CIP were $0.06 \mu \mathrm{g} / \mathrm{ml}$ and $0.015 \mu \mathrm{g} / \mathrm{ml}$, respectively.

\section{Antibiotics and Pharmacokinetics}

We used CTX (Sanofi-Aventis, France) diluted in physiological saline, and a commercial intravenous solution of CIP $(2 \mathrm{mg} / \mathrm{ml}$;
Bayer, France). The dose of CTX administered ( $50 \mathrm{mg} / \mathrm{kg} /$ injection) was identical to that previously reported in an experimental model of neonatal infection. ${ }^{16}$ For CIP, doses were calibrated to achieve a serum concentration within the human therapeutic range 1 to 2 hours after injection. Pharmacokinetic assays were performed in pups as follows. Blood samples were obtained immediately after sacrifice from 3 to 6 pups per group, at selected time points $(30,60,90$, and 120 minutes after subcutaneous antibiotic administration). Sera were conserved at $-20^{\circ} \mathrm{C}$. Antibiotic concentrations were determined by the agar disk diffusion test (microbiological assay), as described by Klassen and Edberg. ${ }^{17}$

As expected, the serum concentration of CTX 1 hour after injection in rat pups, $99.4 \pm 15.2 \mu \mathrm{g} / \mathrm{ml}$, was found to be in the therapeutic range of concentrations usually observed in humans. Among the several doses of CIP tested, a dose of $7.5 \mathrm{mg} / \mathrm{kg} /$ injection was chosen since the peak concentration at 1 hour, $5.5 \pm 0.7 \mu \mathrm{g} / \mathrm{ml}$, was considered to be close to that observed in humans.

\section{Animal Models}

All experimental and animal housing procedures complied with INSERM guidelines and with the Policies on the Use of Animals and Humans in Neuroscience Research.

NEONATAL E. COLI SEPSIS. An E. coli sepsis model was adapted to rat pups from a previously reported study, in order to induce white matter inflammation and damage without bacterial meningitis. ${ }^{18}$ Pathogen-free 4-day-old Sprague-Dawley rat pups were obtained from Charles River Laboratories (France) along with their mothers. At 5 days of age (P5), all pups were inoculated intraperitoneally with $\sim 5,000$ colony-forming units (CFU) of the $\mathrm{C} 5$ strain in physiological saline. Antibiotics were given subcutaneously at 7.5 hours and 24 hours postinfection. These 2 injections were found to be sufficient to sterilize blood culture in infected animals. At several experimental time points (before treatment, 1 hour, 3 hours, and 24 hours after first injection of antibiotics), $5 \mu \mathrm{l}$ of blood was obtained by tail incision and quantitative cultures were performed as previously described. ${ }^{15}$ Cerebrospinal fluid (CSF) samples were obtained as previously described from 20 animals sacrificed 7.5 hours postinfection in order to control the absence of bacterial meningitis. ${ }^{18}$ The detection limit of bacteria in blood was $4 \times 10^{2}$ $\mathrm{CFU} / \mathrm{ml}$. Neonates with negative blood cultures at 7.5 hours postinfection were considered nonbacteremic and were removed from the analysis. Bacteremic animals were sacrificed at P7, P10, and P21 for extensive brain analyses.

GESTATIONAL HYPOXIA. Pregnant Sprague-Dawley rats (Charles River Laboratories, France) were placed in normoxic or hypoxic $\left(10 \% \mathrm{O}_{2}-90 \% \mathrm{~N}_{2}\right)$ gas chambers (Biospherix, Redfield, NY) from embryonic day 5-19 as previously described. ${ }^{19}$ After delivery, normoxic (control) and hypoxic pups given either CTX or CIP treatment on P0 and P1 were studied on P3 and $\mathrm{P} 10$ to assess microglial activation and myelination, respectively. 


\section{Immunohistochemistry}

In each experimental group, we studied at least 6 pups in 3 separate experiments. Immunolabeling with the primary antibody listed in Supporting Table S1 was visualized using the streptavidin-biotin-peroxidase method, as previously described. ${ }^{19,20}$ The Olig2 marker was used to visualize all oligodendrocytes, and adenomatous polyposis coli (APC) and myelin basic protein (MBP) were used to detect postmitotic oligodendrocytes and myelinated fibers, respectively. As previously described, most of the Olig2 nuclei did not colocalize with glial fibrillary acidic protein-positive cells. ${ }^{21}$ Double-labeling was performed with secondary antibodies coupled to the green fluorescent marker Fluoroprobe S488 (Interchim, Montluçon, France) or the red fluorescent marker cyanine 3 (Jackson Immunoresearch Laboratories, West Grove, PA).

\section{Quantitative Measurements}

All quantitative measurements were carried out by observers who were blind to the experimental group of the animal under study.

IMMUNOREACTIVE CELLS. Immunoreactive cells were counted in the white matter underlying the cortex $(+2.16$ to $-0.36 \mathrm{~mm}$ from the bregma). Immunoreactive cells were counted within a $0.065 \mathrm{~mm}^{2}$ grid (at $400 \times$ magnification) in at least 4 sections per animal and at least 6 animals per group at the 2 sacrifice times (P7 and P10).

OPTICAL DENSITY OF MBP-POSITIVE FIBERS The optical density of MBP-immunoreactive fibers was measured in the cingulum in coronal sections $(+2.16$ to $-0.36 \mathrm{~mm}$ from the bregma) as previously reported. ${ }^{20}$ At least 4 sections each from 6-10 animals per group were examined on P10. The optical density was measured at $100 \times$ magnification using a computerized image analysis system (ImageJ, NIH; http://rsb.info. nih.gov/ij) that read optical density as gray levels. Nonspecific background densities were measured at each brain level in a region devoid of MBP immunostaining, and subtracted from values for the cingulum.

\section{Quantitative Real-Time Polymerase Chain Reaction}

DNA-free total RNA from normoxic and hypoxic brain cortices including the white matter was obtained using a protocol adapted from Chomczynski and Sacchi. ${ }^{22}$ The primers for realtime polymerase chain reaction (RT-PCR) were designed using M-fold and Oligo6 software, based on published complementary DNA (cDNA) sequences for genes of interest (see Supporting Table S2). The nature of the amplified DNA was confirmed by sequencing. To standardize gene expression across samples, we used hypoxanthine-guanine phosphoribosyltransferase (HPRT), a housekeeping gene remaining highly stable among the different samples and treatment conditions. For reverse transcription, we used 600ng of total RNA and the Iscript cDNA synthesis kit (Bio-Rad, Marne la Coquette, France). RTPCR was set up using SYBR green-containing supermix (Bio$\mathrm{Rad})$ for 50 cycles with a 3 -step program (25-second denatura- tion at $96^{\circ} \mathrm{C}, 30$-second annealing at $60^{\circ} \mathrm{C}$, and 30 -second extension at $72^{\circ} \mathrm{C}$ ). Amplification specificity was assessed by melting curve analyses. Each experiment was run twice with at least 6 animals per group, and in both cases measurements were carried out in triplicate.

\section{Luminex Analysis of Cytokine Concentrations}

Serum concentrations of 8 cytokines were measured using the Luminex xMAP technique (multianalytic profiling) according to the manufacturer's guidelines (Bio-Rad). The detection and quantification of cytokine levels was performed using a BioPlex 200 system (Bio-Rad). Analysis of data was performed using Bio-Plex Manager 5.0 software (Bio-Rad).

\section{Statistical Analysis}

All data were reported as means \pm standard error (SEM). Analysis of variance was performed with age and group (uninfected, CTX-treated, or CIP-treated animals) as factors, and the Newman-Keuls post-hoc nonparametric test was used. Statistical tests were run on GraphPad Prism version 4.00 (GraphPad Software, San Diego, CA).

\section{Results}

Establishment of an E. coli Sepsis Model and Curative Antibiotic Treatments in Neonatal Rats In our animal model characterized by perinatal $E$. coli sepsis, bacteremia was detected in 70 of 76 (92\%) P5 animals 7.5 hours after $E$. coli inoculation, and ranged from $4.8 \times 10^{3}$ to $3.2 \times 10^{5} \mathrm{CFU} / \mathrm{ml}$. By sampling CSF from 20 infected pups, we confirmed that none of the animals with bacteremia had meningitis at this early time point. Antibiotic treatment was associated with a high rate of cure. We performed quantitative cultures of blood samples at 1 and 3 hours after the first dose of antibiotics. No difference in bacterial clearance has been observed between the 2 antibiotics (Supporting Fig S1). Only 1 in 37 and 5 in 39 neonatal rats died 24 hours after treatment with CIP and CTX, respectively. In the survivors, bacteremia was no longer detectable after 24 hours of treatment. In contrast, $100 \%$ of rat pups with bacteremia died within 24 hours in the absence of antibiotic treatment.

\section{E. coli Sepsis Induces an Inflammatory Response in the Developing White Matter}

The consequences of $E$. coli sepsis in the developing CNS were first investigated by assessing microglial activation throughout the developing white matter. We compared at P7 and P10 sham-infected pups and infected pups treated either with the reference antibiotic CTX or with CIP. Neither CTX nor CIP induced altered microglial activation, morphology, or density in sham-infected pups compared to untreated sham-infected pups. E. coli 
A

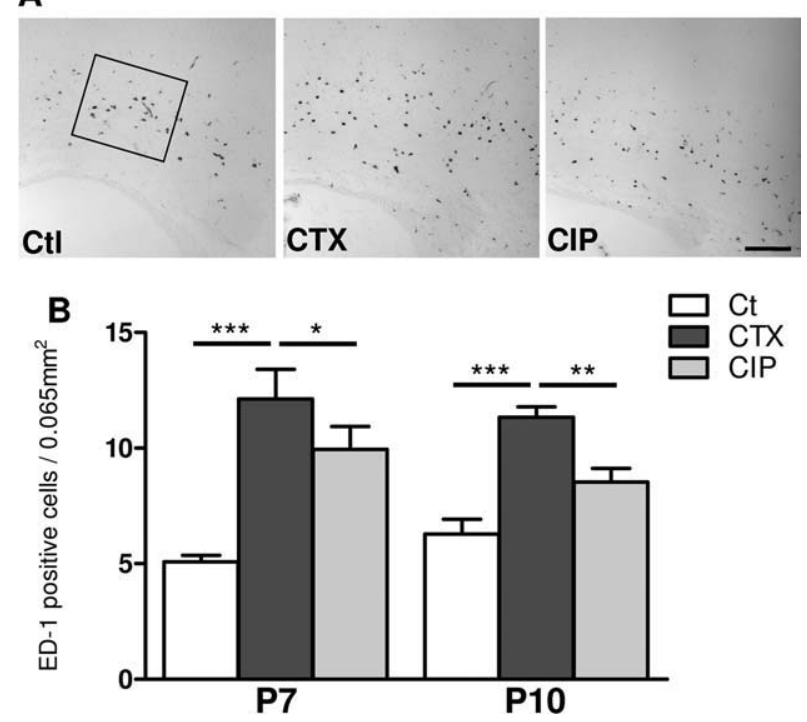

FIGURE 1: E. coli sepsis induces a white matter inflammatory response. (A) ED1-immunoreactivity in the cingulate white matter of P7 rat pups demonstrating that the density of activated microglia was dramatically increased in infected animals compared to sham infected control (Ct). CTX-treated infected rat pups exhibit higher activated microglial densities than CIP-treated infected animals. Bars $=200 \mu \mathrm{m}$. (B) Quantitative analysis of ED1positive cells in the cingulate white matter of sham-infected control $(\mathrm{Ct})$ pups and infected CTX-treated or CIP-treated animals at P7 ( $n=11-15)$ and P10 ( $n=8-10)$. ${ }^{*} p<0.05$, ${ }^{\star \star} p<0.01$, and ${ }^{* \star} p<0.001$, using 1 -way ANOVA with the Newman-Keuls correction. ANOVA = analysis of variance.

sepsis was associated with a dramatic increase in the density of activated microglia in the white matter of infected rat pups (Fig 1A, B). However, activated microglial density was found to be significantly lower in CIP-treated animals than in CTX-treated pups $(p<0.05)$. This observation did not appear to be related to a difference in bacteremia between the 2 groups at any time points investigated (Supporting Fig S1). Indeed, before antibiotic treatment was initiated, bacterial concentrations were similar in rat pups that received CTX and in those that received CIP $(4.65 \pm 0.45 \mathrm{log} \mathrm{CFU} / \mathrm{ml}$ vs $4.84 \pm 0.79$ $\log \mathrm{CFU} / \mathrm{ml} ; p>0.05)$. No difference in bacterial clearance has been observed between the 2 antibiotics 1 hour and 3 hours after the first dose of antibiotics. Bacteremia was no longer detectable after 24 hours of treatment.

\section{Cytokine Production in Response to E. coli Sepsis in Blood and CNS Compartments}

Because microglial activation in the CNS could be related in our model to systemic inflammation, we next explored the impact of systemic E. coli sepsis on cytokine production in rat pups (Fig 2). Before antibiotic treatment (7.5 hours after inoculation), the concentrations of several proinflammatory cytokines (including interleukin
[IL]-1 $\beta$, IL-6, and tumor necrosis factor [TNF]- $\alpha$ ) were found to be increased in infected pups when compared to sham-infected controls (see Fig 2A). IL-10 and interferon $-\gamma$ was also increased, whereas the serum concentration of IL-4 was decreased in response to E. coli sepsis (data not shown). Twenty-four hours after antibiotic initiation, both CTX and CIP were associated with a similar decrease in proinflammatory cytokine concentrations in infected rat pups. Similarly, proinflammatory cytokine gene expression was found to be increased in brain tissue in response to E. coli sepsis. Again, antibiotics were able to reverse this overexpression but no difference was detectable between CTX and CIP (see Fig 2B).

Because reduction in activated microglia density in the CIP group was not associated with detectable cytokine production modulation, we next investigated inducible NO synthase, 1 of the major components of microglial activation. Using immunocytochemistry, we found that E. coli sepsis significantly induced iNOS expression in P10 rat pups. Compared to CTX treatment, CIP significantly reduced this induction in infected animals (Fig $3 \mathrm{~A}-\mathrm{D}$ ). As expected, all iNOS-positive cells colocalized with microglial cells labeled using tomato lectin (see Fig 3E).

\section{E. coli Sepsis Is Associated with Defective Myelination}

We next investigated the impact of neonatal sepsisinduced microglial activation on myelination of the developing white matter. Neither CTX nor CIP had any effect on myelination in the developing white matter of sham-infected P10 and P21 rat pups. Infected animals treated with the reference antibiotic CTX demonstrated altered myelin content in the lateral corpus callosum (Fig 4A). The density of MBP in these animals was found to be $40 \%$ lower than in uninfected controls on P10. However, this phenomenon was transient, as no further differences were observed on P21. This myelination delay was independent of the brain area considered, and was observed in both the cingulate white matter and the genu of the corpus callosum. Compared to CTX treatment, CIP significantly reduced the occurrence of a myelination delay in infected animals. CIP-treated animals exhibited a density of MBP similar to that of uninfected pups (see Fig 4B). These findings were all independent of gender.

\section{CIP Enhances Oligodendroglial Maturation and Survival in Neonatal Rats Subjected to E. coli Sepsis}

We next asked the question of whether the deficient myelination observed in infected pups could be related to a modulation of oligodendroglial cell death or maturation. We compared the density of the total oligodendroglial 


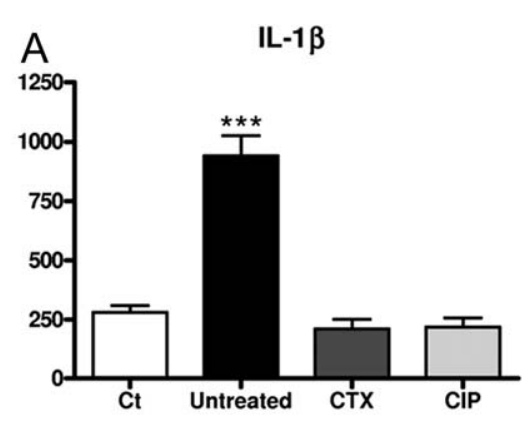

IL-1 $\beta$

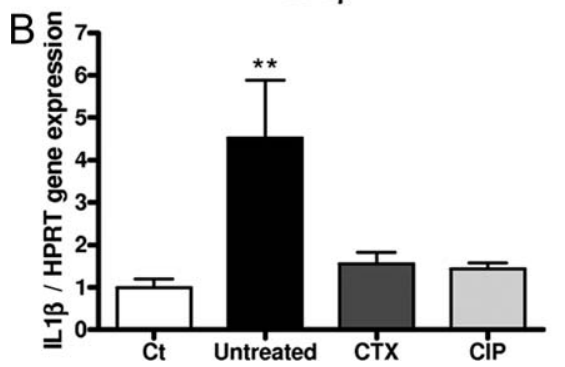

IL-6

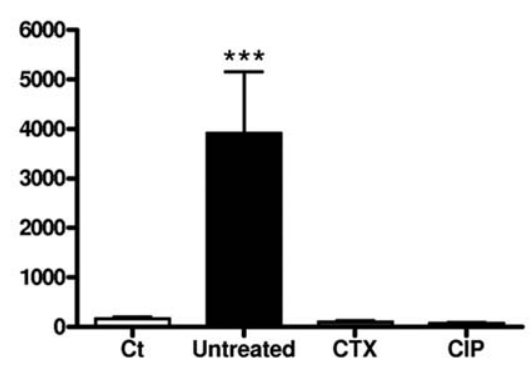

IL-6

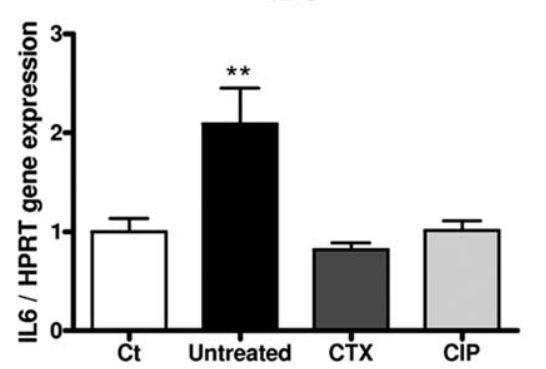

TNF- $\alpha$

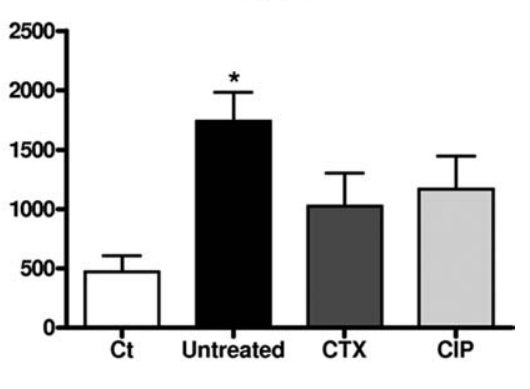

TNF- $\alpha$

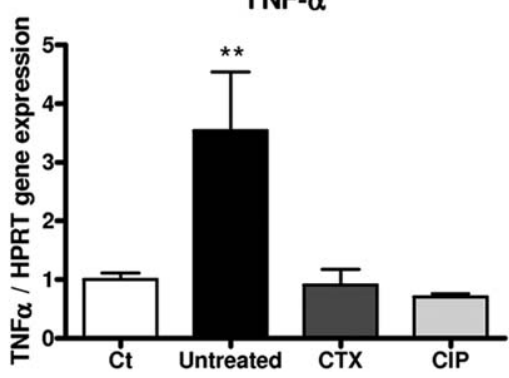

FIGURE 2: Inflammatory response to $E$. coli sepsis in blood and CNS compartments. (A) LUMINEX multiplex assay for serum cytokines in uninfected controls $(C t, n=6)$, infected untreated animals 7.5 hours after $E$. coli inoculation $(n=10)$, and infected animals 24 hours after antibiotic treatment (CTX, $n=13$; or CIP, $n=14)$. IL-1 $\beta$, IL-6, and TNF- $\alpha$ concentrations are reported in $\mathrm{pg} / \mathrm{ml}$. ${ }^{*} \mathrm{p}<0.05$ and ${ }^{* * *} \mathrm{p}<0.001$ using 1-way ANOVA with the Newman-Keuls correction to compare untreated and antibiotictreated infected animals with sham-infected controls. (B) Quantitative analysis of IL-1 $\beta$, IL-6, and TNF- $\alpha$ gene expression (quantitative RT-PCR) in sham-infected animals $(C t, n=8)$ compared to infected animals just before antibiotic treatment $(n=7)$ and infected animals 24 hours after the initiation of treatment with CTX $(n=6)$ or CIP $(n=8)$. ${ }^{*} p<0.05$ and ${ }^{* * \star} p<0.001$ using 1 -way ANOVA with the Newman-Keuls correction to compare infected groups with controls. ANOVA = analysis of variance.

population (olig2+ cells) within the white matter of control and infected P10 rat pups treated with either CTX or CIP. E. coli sepsis was associated with a marked decrease in the density of olig2 + oligodendrocytes in CTX-treated animals in the lateral corpus callosum (Fig 5A). In contrast, CIP was able to significantly prevent this decrease (see Fig 5A). Consistent with these observations, the increase in oligodendroglial cell death (the density of olig2/terminal deoxynucleotidyl transferase-mediated deoxyuridine triphosphate nickend labeling [TUNEL] double-positive cells) observed in CTX-treated rat pups when compared to controls was not seen in CIP-treated animals (see Fig 5B).

The density of mature oligodendrocytes (APC+ cells) was found to be lower in CTX-treated but not in CIP-treated rat pups compared to uninfected animals on P10 (see Fig 5C). Concordantly, CTX-treated animals exhibited a significantly higher density of olig2/Ki67 double-positive cells than either uninfected or CIPtreated animals (see Fig 5D). Finally, the density of mature oligodendrocytes (APC+ cells) among olig2+ oligodendrocytes was found to be higher in CIP-treated compared to CTX-treated rat pups on P10 (see Fig 5E). These results suggest that CIP treatment reduced the oligodendroglial cell death observed in infected pups treated with CTX, and led to the conservation of oligodendroglial maturation in the injured white matter.
We next explored the impact of antibiotic treatment on the relative expression of semaphorin $3 \mathrm{~A}$ and $3 \mathrm{~F}$, which are involved in the myelination of axons in the CNS. Semaphorin $3 \mathrm{~A}$ and $3 \mathrm{~F}$ are known to act as axonal guidance cues and chemotactic factors for oligodendroglial cells in the developing CNS. ${ }^{23,24}$ Semaphorin $3 \mathrm{~A}$ is associated with a repulsive signal and semaphorin $3 \mathrm{~F}$ with an attractive signal toward migrating oligodendrocyte precursor cells. In our study, E. coli sepsis was associated with a remarkable upregulation of semaphorin $3 \mathrm{~A}$ and downregulation of semaphorin 3F expression (Fig 6A,B). Interestingly, CIP and CTX treatments resulted in the differential regulation of the transcription of these 2 genes: CTX only partially attenuated the effects of E. coli sepsis, whereas CIP completely reversed them. These data suggest that CIP could have a specific impact on the expression of the guidance molecules semaphorin $3 \mathrm{~A}$ and $3 \mathrm{~F}$, and may thus determine the ability of injured white matter to remyelinate.

\section{CIP Prevents Myelination Deficits in a Noninfectious Rat Model of WMD}

We hypothesized that the CIP-related neuroprotection observed in our E. coli sepsis model could be due to its intrinsic anti-inflammatory properties and not to its antibacterial activity. To test this hypothesis, we used both CIP and CTX (2 subcutaneous injections on P0-P1) in another 

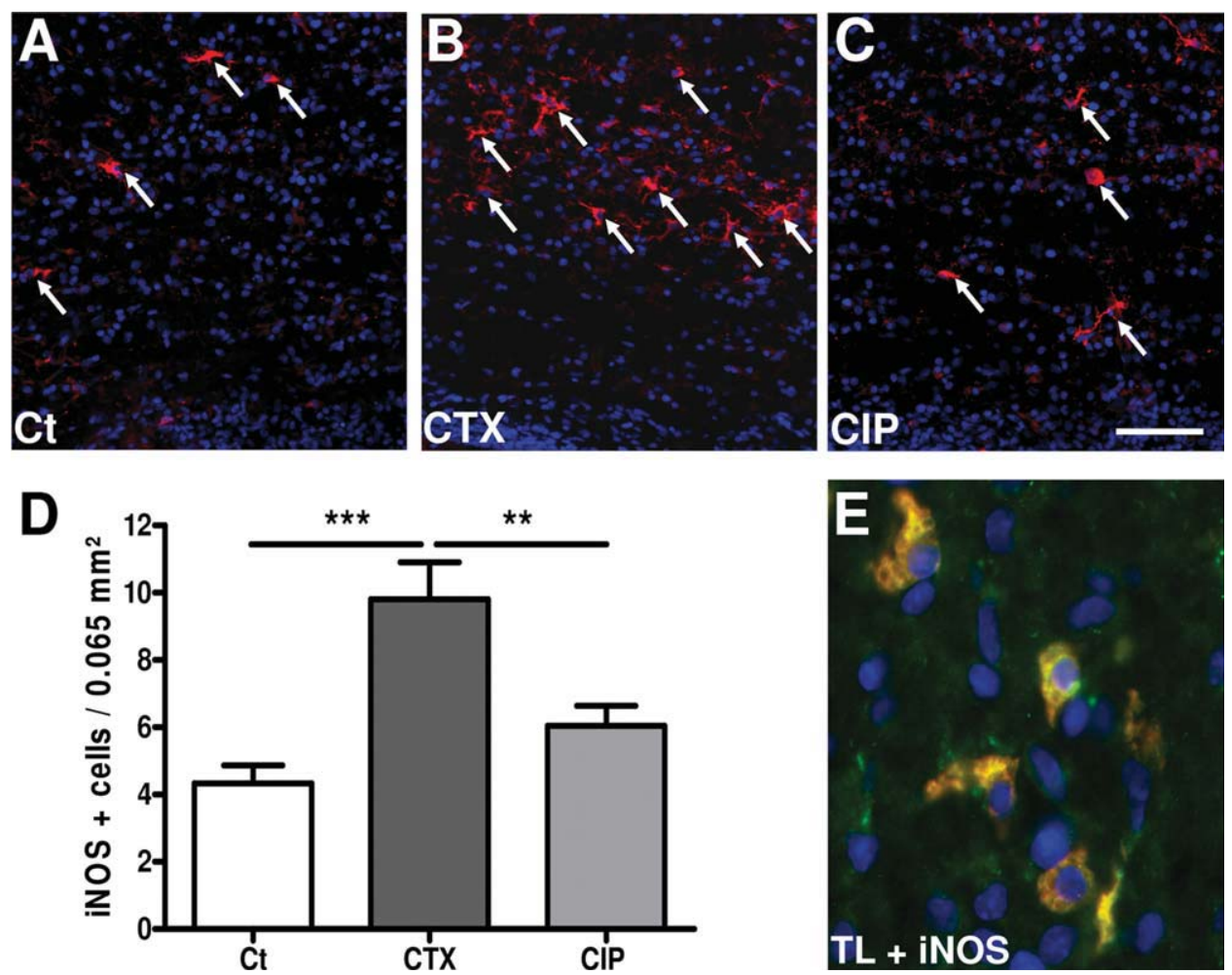

FIGURE 3: CIP prevents iNOS overexpression in the developing white matter subjected to E. coli sepsis. (A-C) iNOSimmunoreactivity in the cingulate white matter of P10 rat pups demonstrating that the density of iNOS-positive cells was increased in infected animals compared to sham-infected controls $(C t, n=8)$. CTX-treated infected rat pups $(n=6)$ exhibit higher iNOS-positive cell density than CIP-treated infected animals $(n=8)$. Bars $=200 \mu \mathrm{m}$. (D) Quantitative analysis of iNOSpositive cells in the cingulate white matter of sham-infected controls $(\mathrm{Ct}, \mathrm{n}=8)$ pups and infected CTX-treated $(n=6)$ or CIPtreated $(n=8)$ animals. ${ }^{* *} p<0.01$ and ${ }^{* * *} p<0.001$, using 1 -way ANOVA with the Newman-Keuls correction. (E) Double immunolabeling using tomato lectin (red) and iNOS (green), demonstrating that all iNOS-positive cells colocalized with microglial cells in the cingulate white matter of P10 infected CTX-treated rat pups. ANOVA = analysis of variance.

rat model of WMD, characterized by white matter inflammation induced by gestational hypoxia. ${ }^{19}$ In this model, microglial activation leads to myelination delays with similar features to those described in the E. coli model investigated above. CTX was found to be unable to modulate either microglial activation at $\mathrm{P} 3$ or the myelination deficit at P10 observed after gestational hypoxia. In contrast, CIP fully reduced the microglial activation observed in the white matter of pups born after gestational hypoxia (Fig 7A). Similarly, CIP restored MBP content (see Fig 7B) and was associated with a mature oligodendrocyte density similar to that of controls (see Fig 7C). These data strongly support the hypothesis that an intrinsic anti-inflammatory property of CIP could explain, at least in part, its effects on the CNS of animals subjected to either gestational hypoxia or $E$. coli sepsis.

\section{Discussion}

In this study, we developed a rat model of neonatal E. coli sepsis without meningitis that induces marked inflammation in the developing white matter. This model is relevant in light of recent clinical studies demonstrating that postnatal sepsis accounts for a large proportion of WMD in very preterm infants. ${ }^{5,25}$ Our animal model, developed at P5, closely mimics the pathological features observed in infected preterm infants, including white matter inflammation and myelination delay. These alterations occur at a developmental stage of the rat brain that corresponds to the human brain at 28-32 weeks of gestation, ${ }^{26}$ recognized as the period during which the developing brain is most vulnerable to either hypoxic or inflammatory insults. ${ }^{27}$

Lipopolysaccharide (LPS) has been previously used to mimic perinatal bacterial infection and to study the consequences of inflammation in the developing brain. ${ }^{3}$ However, although LPS is a major bacterial determinant in the activation of the innate immune system, models using this component may not reflect the complexity of Gram-negative sepsis and its subsequent treatment with antibiotics. E. coli per se has been used to induce brain damage in both antenatal rabbit and rodent models. ${ }^{28-30}$ To our knowledge, our model is the first to explore the effects of postnatal sepsis and antibiotic treatment on the developing brain.

Several lines of evidence support the relationship between E. coli sepsis and WMD in our model. First, postnatal E. coli sepsis induces hypomyelination of the 


\section{A}
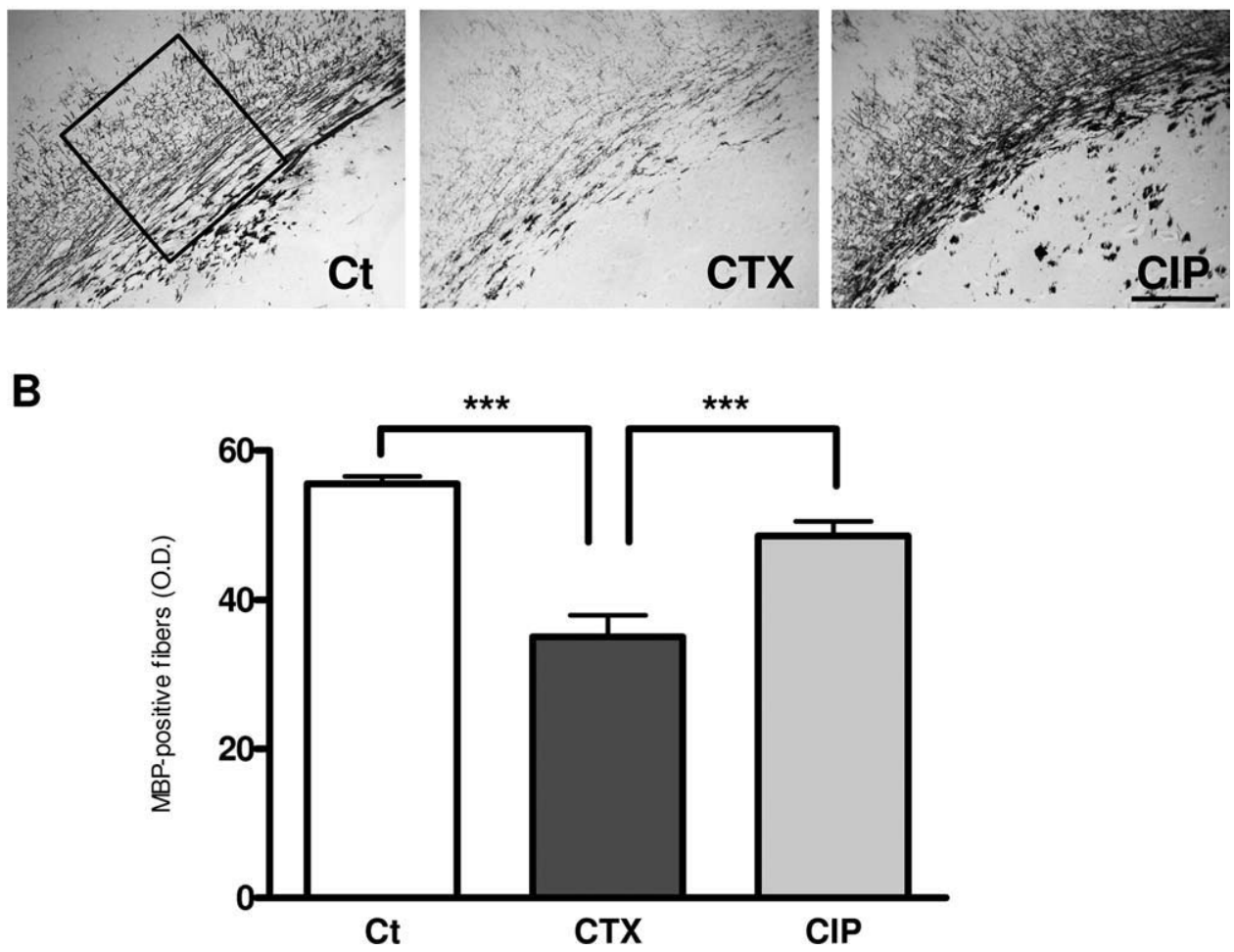

FIGURE 4: E. coli sepsis induces myelination deficits in the developing white matter. (A) MBP-immunoreactivity in the cingulate white matter of P10 rat pups demonstrating that myelin content is deficient in CTX-treated animals compared to their CIPtreated counterparts or uninfected controls $(\mathrm{Ct})$. Bar $=200 \mu \mathrm{m}$. B: Quantitative analysis (optical density) of MBP-positive fibers in the cingulate white matter of controls $(C t, n=14)$ and infected CTX-treated $(n=15)$ and CIP-treated animals $(n=13)$. The area over which optical density was measured is indicated by the square in $A .{ }^{\star \star \star} p<0.001$ using one-way ANOVA with the Newman-Keuls correction. ANOVA = analysis of variance.

developing white matter. Several experimental studies have demonstrated a similar effect of LPS given antenatally to rabbits and sheep. ${ }^{30,31}$ Second, we have also observed that E. coli sepsis induces an increase in oligodendroglial cell death in the developing white matter. This finding is consistent with recent data demonstrating that immature oligodendrocytes are highly vulnerable to both inflammation and oxidative stress. ${ }^{27,32-35}$ Finally, we found here that $E$. coli sepsis induced increased serum concentrations of TNF$\alpha$ and IFN- $\gamma$ that have been shown to block the differentiation of oligodendrocyte precursors in vitro. ${ }^{36,37}$

Interestingly, our model has unveiled a new pathophysiological consequence of E. coli sepsis, with the specific regulation of semaphorin $3 \mathrm{~A}$ and $3 \mathrm{~F}$. A recent report has demonstrated that semaphorin $3 \mathrm{~A}$ (repulsive) and $3 \mathrm{~F}$ (attractive) are involved in the control of oligodendrocyte precursor cell migration in multiple sclerosis, and hence may determine the ability of plaques to remyelinate..$^{23,24}$ In demyelinating diseases, remyelination could fail either because oligodendroglial progenitors fail to repopulate areas of demyelination, or because they are unable to generate remyelinating oligodendrocytes in the presence of persistent inflammation. ${ }^{38}$
Taken together, our results suggest that the E. coli sepsis model is relevant to WMD, being able to account for myelination delay through several pathways including increased oligodendroglial cell death, impairment of oligodendroglial maturation, imbalance in axonal guidance, and reduced attraction of axons to myelin sheets, and finally, impaired regeneration of injured white matter areas. We therefore used this model to assess the consequences of treatment with 2 antibiotics in WMD.

Our comparative study of CTX and CIP strongly suggests that CIP exerts a neuroprotective effect in our model of E. coli sepsis-induced WMD, probably through the modulation of white matter inflammation. Several in vitro studies but only a few reports using in vivo models with living microorganisms showed that fluoroquinolones, including CIP, inhibit the synthesis of proinflammatory cytokines such as TNF- $\alpha$, IL- $1 \beta$, and IL- $6 .{ }^{13}$ In most of the in vitro previous studies, concentrations of fluoroquinolones were much higher than those observed in clinical setting. Here, we failed to demonstrate that CIP induced inhibition in the synthesis of proinflammatory cytokines in vivo. The lower (and clinically relevant) antibiotic concentrations used in our model may explain this discrepancy at 
A
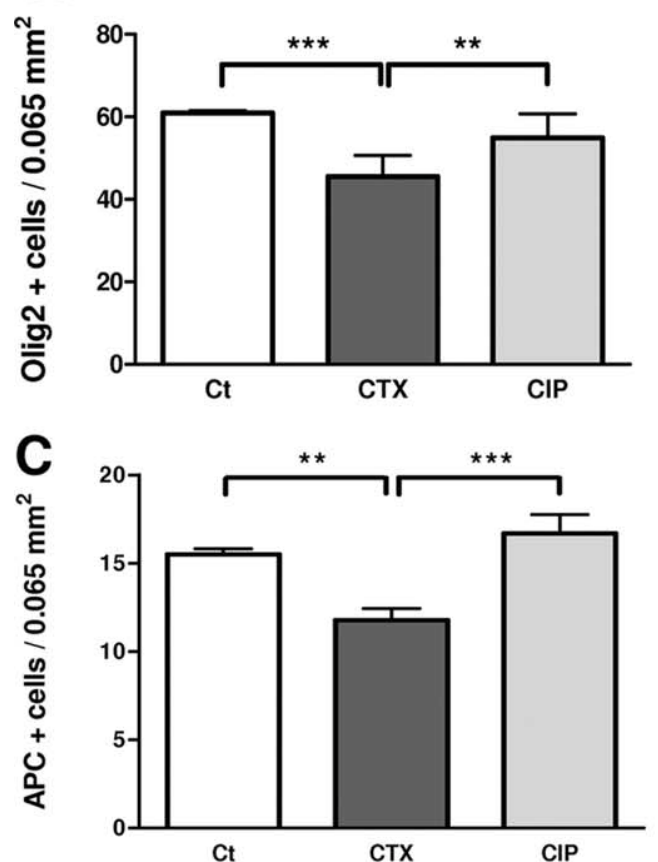

E
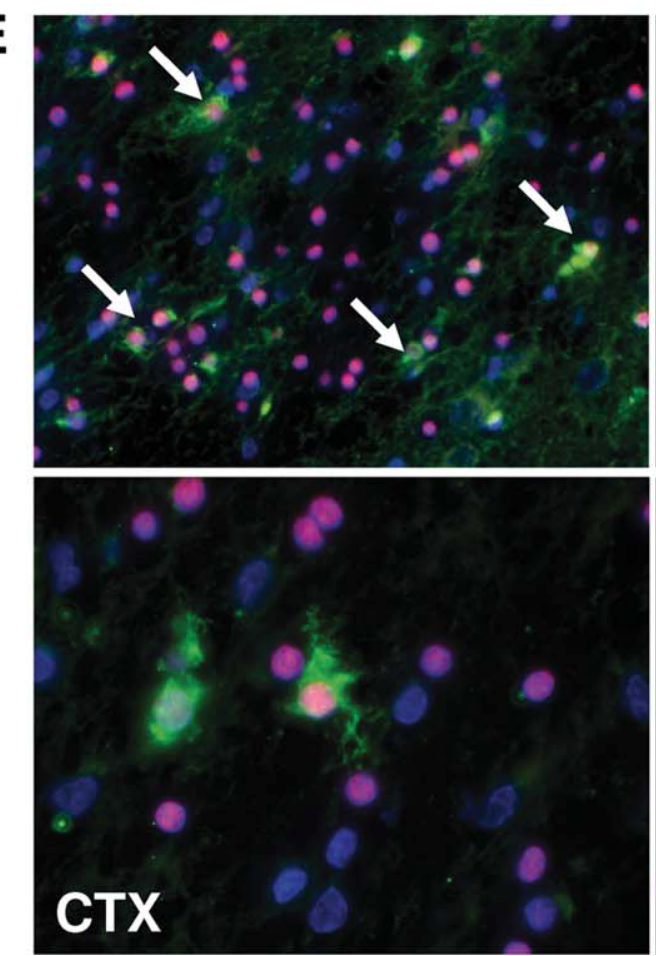

B

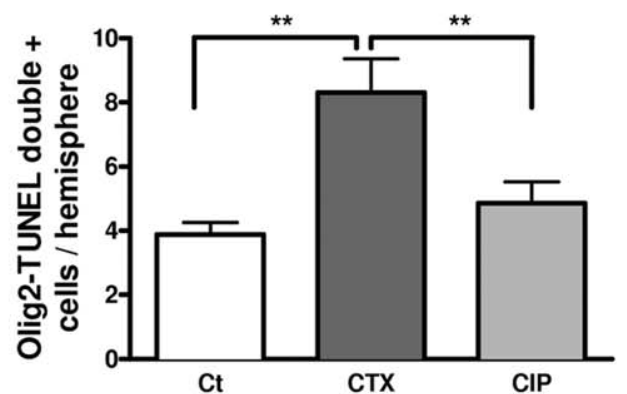

D
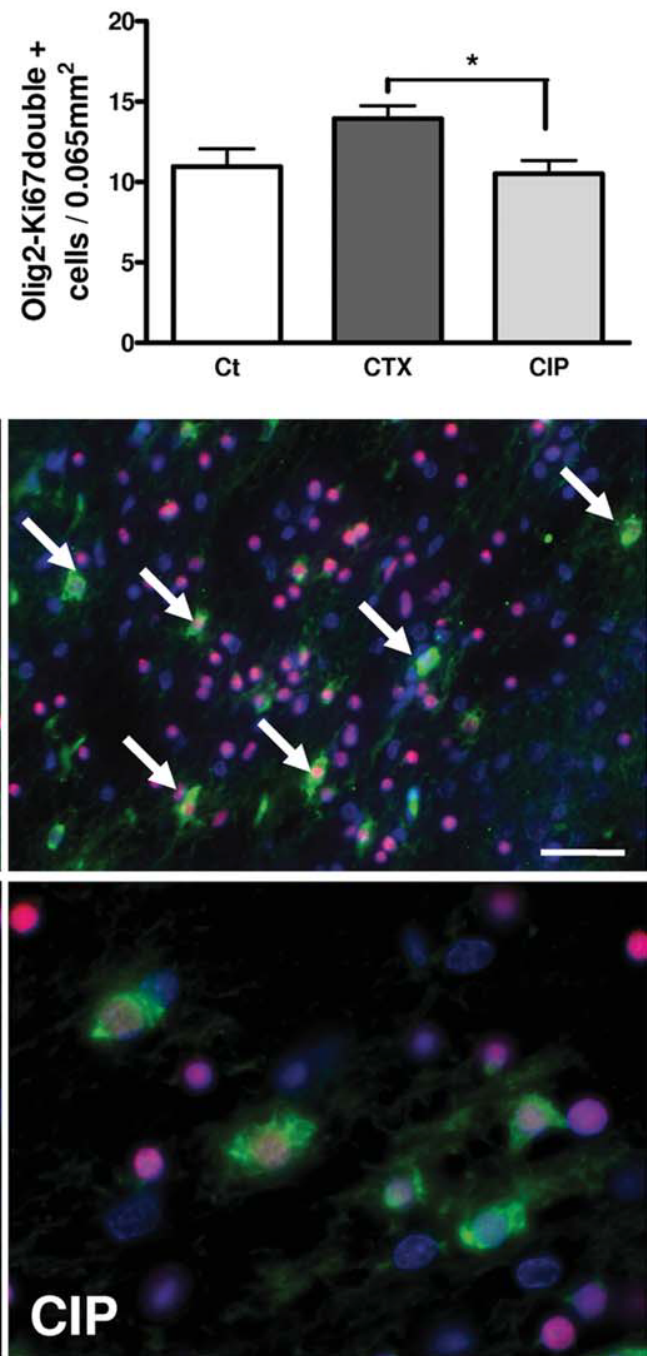

FIGURE 5: CIP enhances oligodendroglial maturation and survival in neonatal rats subjected to E. coli sepsis. (A) Quantitative analysis of Olig2-immunoreactive cells in the cingulate white matter at P10. CIP treatment reverses the loss of mature oligodendrocytes observed in infected CTX-treated pups. ${ }^{* \star} p<0.01$ and ${ }^{* \star *} p<0.001$, using 1-way ANOVA with the NewmanKeuls correction, $\mathrm{n}=6$ for each experimental groups. (B) Quantification of TUNEL+ oligodendrocytes (Olig2+) in the hemispheric white matter. CIP treatment prevents the increase in oligodendrocyte cell death observed in infected CTX-treated pups. ${ }^{\star \star} p<0.01$ using 1-way ANOVA with the Newman-Keuls correction, $n=6$ for each experimental group. (C) Quantitative analysis of APCimmunoreactive cells in the cingulate white matter at P10. CIP treatment reverses the loss of mature oligodendrocytes observed in infected CTX-treated pups. ${ }^{* *} p<0.01$ and ${ }^{* \star *} p<0.001$, using 1-way ANOVA with the Newman-Keuls correction, $n=10-13$ for each experimental group. (D) Quantitative analysis of the Ki67/Olig2-positive cells in the cingulate white matter of controls (Ct, $n=8)$ and infected CTX-treated $(n=12)$ and CIP-treated $(n=10)$ animals. ${ }^{*} p<0.05$ using 1-way ANOVA with the Newman-Keuls correction. (E) APC/Olig2-immunoreactivity in the cingulate white matter of P10 rat pups, demonstrating the increase in the density of double-labeled oligodendrocytes (mature; arrows) after CIP treatment. Bar $=100 \mu \mathrm{m}$. ANOVA = analysis of variance. 

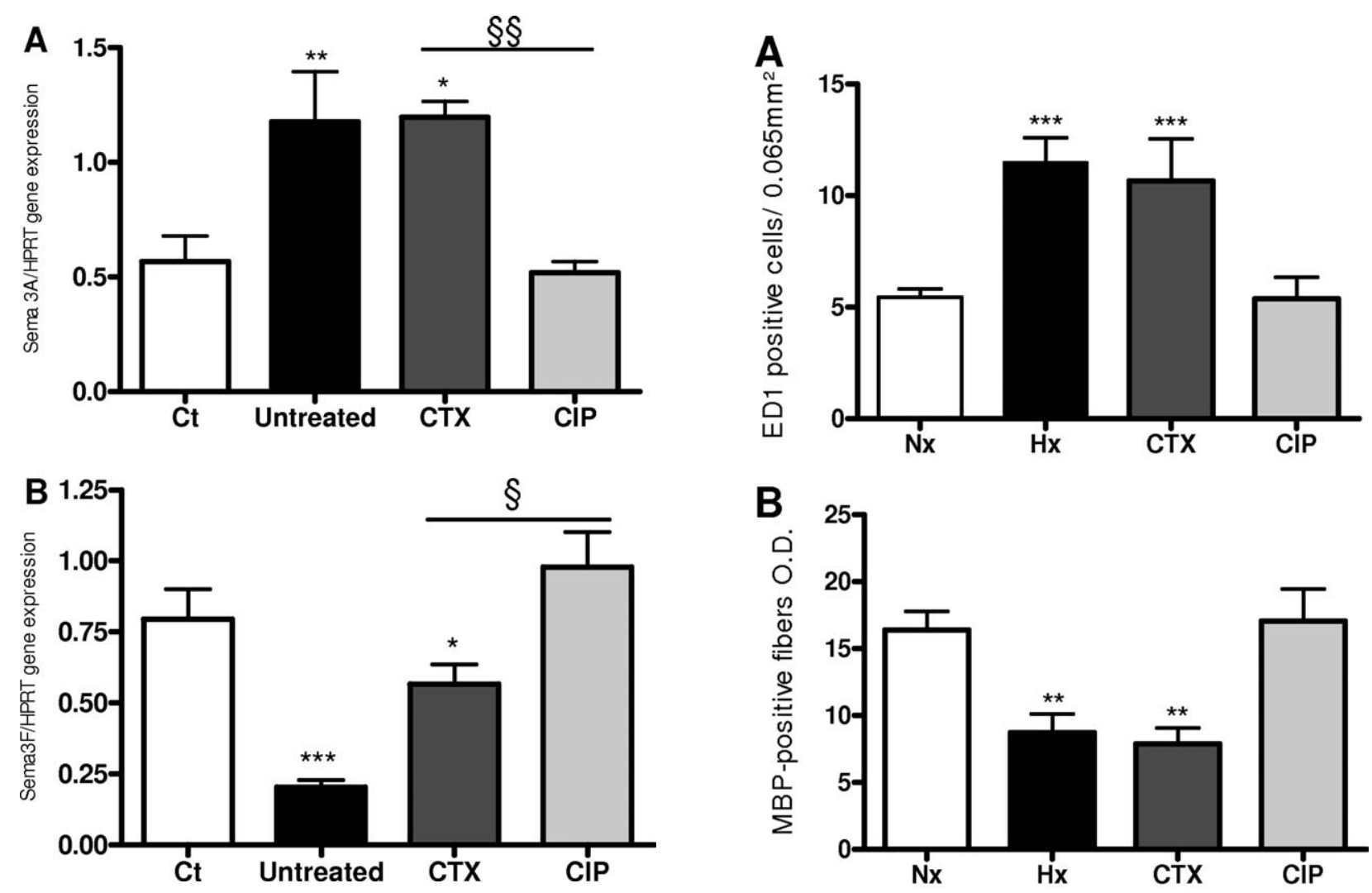

FIGURE 6: Quantitative analysis of semaphorin $3 \mathrm{~A}$ and $3 \mathrm{~F}$ gene expression. Quantitative analysis of gene expression of (A) semaphorin $3 \mathrm{~A}$ (Sema3A) and (B) semaphorin 3F (Sema3F) in sham-infected animals $(\mathrm{Ct}, \mathrm{n}=8$ ) compared to infected animals just before antibiotic treatment $(n=7)$ and infected animals 24 hours after the initiation of treatment with CTX $(n=6)$ or CIP $(n=8)$. ${ }^{*} p<0.05$ and ${ }^{* * *} p<0.001$ using 1-way ANOVA with the Newman-Keuls correction to compare infected groups with controls. ${ } p<0.05$ and $\S_{p} p<$ 0.01 using 1-way ANOVA with the Newman-Keuls correction to compare CTX-treated and CIP-treated animals. ANOVA $=$ analysis of variance.

least in part. Conversely, we found that CIP was able to significantly reduce the iNOS overexpression induced by E. coli sepsis in microglial cells. Intense iNOS expression occurred in activated microglia during the acute stage of WMD in humans. ${ }^{39}$ Therefore, inhibition of iNOSinduced nitrosative stress by CIP could have beneficial effect in the injured developing white matter.

In the literature, several initial targets have been suggested to explain the anti-inflammatory property of the fluoroquinolones: (1) the inhibitory effect of phosphodiesterase and the subsequent intracellular accumulation of cyclic adenosine monophosphate and activation of protein kinase C; (2) the increase in the gene transcription of nuclear factor of activated T cells (NFAT-1), activator protein-1 (AP-1), and nuclear factor IL-2A ${ }^{40,41}$; (3) the inhibition of nuclear factor kappa B activation ${ }^{42}$; and (4) the decrease in the expression of the LPS

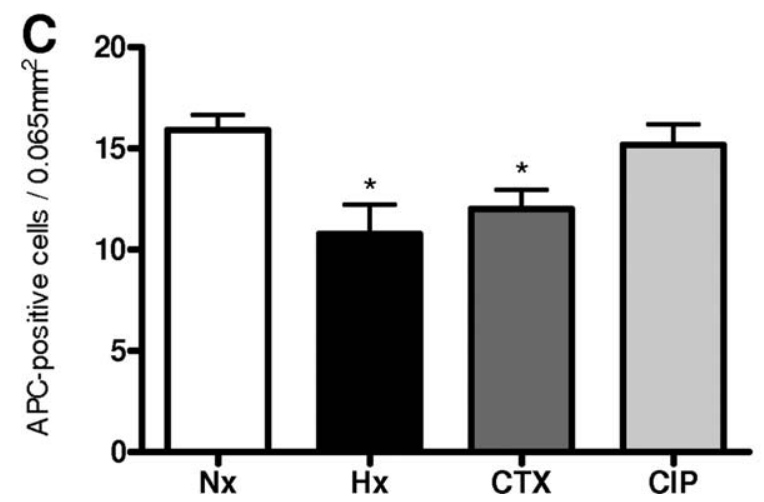

FIGURE 7: CIP prevents myelination deficits in a noninfectious rat model of WMD. (A) Quantitative analysis of ED1-positive cells in the cingulate white matter of normoxic pups $(\mathrm{Nx}, \mathrm{n}=15)$ in comparison to either untreated hypoxic $(\mathrm{Hx}, \mathrm{n}=6)$ or CTX-treated $(\mathrm{n}=8)$ or CIP-treated $(n=8)$ hypoxic animals at P3. ${ }^{* \star} p<0.001$ using 1-way ANOVA with the Newman-Keuls correction to compare hypoxic animals with normoxic controls. (B) Quantitative analysis (optical density) of MBP-positive fibers in the cingulate white matter of normoxic pups $(\mathrm{Nx}, \mathrm{n}=10)$ in comparison to either untreated hypoxic $(\mathrm{Hx}, \mathrm{n}=6)$ or CTX-treated $(n=8)$ or CIP-treated $(n=8)$ hypoxic animals at P10. ${ }^{* *} p<0.01$ using 1-way ANOVA with the NewmanKeuls correction to compare hypoxic animals with normoxic controls. (C) Quantitative analysis of APC-positive mature oligodendrocytes in the cingulate white matter of normoxic $(\mathrm{Nx}, \mathrm{n}=10)$ pups in compared to either untreated hypoxic $(\mathrm{Hx}, \mathrm{n}=6)$ or CTX-treated $(n=8)$ or CIP-treated $(n=8)$ hypoxic animals at P10. ${ }^{*} p<0.05$ using 1 -way ANOVA with the Newman-Keuls correction to compare hypoxic animals with normoxic controls. ANOVA $=$ analysis of variance. 
receptor complex. ${ }^{43}$ These particular properties seem to be shared only by fluoroquinolones harboring a cyclopropyl group at the N1 position or a piperazinyl group at the C7 position. ${ }^{13,44}$ Interestingly, CIP is particular among fluoroquinolones, as both residues are present on this molecule. To date, the clinical relevance of inflammatory modulation by the fluoroquinolones remains to be determined. Indeed, in human studies, the clinical benefits of such an effect are still uncertain. Gogos and colleagues $^{45}$ have shown that CIP attenuates the proinflammatory response as compared to cephalosporins, in patients with Gram-negative sepsis. However, this difference has no impact on the outcome. ${ }^{45}$

In premature neonates, E. coli is the major cause of early onset sepsis. ${ }^{7}$ The molecule of choice to treat such infections is a third-generation cephalosporin such as CTX. Although this antibiotic is highly effective against E. coli, beta-lactam cell wall activity may cause the release of cell wall components such as LPS, which can lead to an excessive inflammatory response. Fluoroquinolones that do not target bacterial membranes might induce a lower release of LPS and subsequently, the lower expression of proinflammatory cytokines than cephalosporins. ${ }^{18}$ However, the effect of CIP on endotoxin release remains controversial. ${ }^{46}$ In our model, we failed to demonstrate any difference in systemic cytokine patterns induced by the 2 antibiotics. We could thus speculate that the neuroprotection afforded by CIP is likely due to a direct effect on the developing CNS.

It remains to be determined if CIP exerts its neuroprotective effect by counteracting the effects of systemic cytokines on the CNS, or by preventing the deleterious effects of hypoxia, consequently to sepsis.

In conclusion, this study provides strong evidence for a novel neuroprotective property of ciprofloxacin in neonatal rat models of inflammation-induced brain lesions possibly related to modulation of iNOS expression in the developing white matter. The impact of CIP needs to be further explored in other perinatal inflammatory conditions such as chorioamnionitis and necrotizing enterocolitis. Delineating the molecular determinants of the neuroprotective effects of CIP could lead to the design of potential new candidates for the prevention of noninfectious WMD or the enhancement of myelin repair.

\section{Authorship}

S.B. and O.B. contributed equally to this work.

\section{Potential Conflicts of Interest}

Nothing to report.

\section{References}

1. Larroque B, Ancel PY, Marret S, et al. Neurodevelopmental disabilities and special care of 5-year-old children born before 33 weeks of gestation (the EPIPAGE study): a longitudinal cohort study. Lancet 2008;371:813-820.

2. Marlow N, Wolke D, Bracewell MA, Samara M; EPICure Study Group. Neurologic and developmental disability at six years of age after extremely preterm birth. N Engl J Med 2005;352:9-19.

3. Hagberg $H$, Peebles D, Mallard C. Models of white matter injury: comparison of infectious, hypoxic-ischemic, and excitotoxic insults. Ment Retard Dev Disabil Res Rev 2002;8:30-38.

4. Dammann $\mathrm{O}, \mathrm{O}^{\prime}$ Shea TM. Cytokines and perinatal brain damage. Clin Perinatol 2008;35:643-663.

5. Shah DK, Doyle LW, Anderson PJ, et al. Adverse neurodevelopment in preterm infants with postnatal sepsis or necrotizing enterocolitis is mediated by white matter abnormalities on magnetic resonance imaging at term. J Pediatr 2008;153:170-175.

6. Volpe JJ. Postnatal sepsis, necrotizing enterocolitis, and the critical role of systemic inflammation in white matter injury in premature infants. J Pediatr 2008;153:160-163.

7. Stoll BJ, Hansen NI, Higgins RD, et al. Very low birth weight preterm infants with early onset neonatal sepsis: the predominance of gram-negative infections continues in the National Institute of Child Health and Human Development Neonatal Research Network, 2002-2003. Pediatr Infect Dis J 2005;24:635-639.

8. Bizzarro MJ, Dembry LM, Baltimore RS, Gallagher PG. Changing patterns in neonatal Escherichia coli sepsis and ampicillin resistance in the era of intrapartum antibiotic prophylaxis. Pediatrics 2008;121: 689-696.

9. Neijens HJ. Ciprofloxacin in preterm neonates: case report and review of the literature. Eur J Pediatr 1998;157:843-845.

10. Houdouin V, Bonacorsi S, Bidet $\mathrm{P}$, et al. Association between mortality of Escherichia coli meningitis in young infants and non-virulent clonal groups of strains. Clin Microbiol Infect 2008;14:685-690.

11. Wispelwey B. Clinical implications of pharmacokinetics and pharmacodynamics of fluoroquinolones. Clin Infect Dis 2005;41:S127-S235.

12. Dalhoff A, Shalit I. Immunomodulatory effects of quinolones. Lancet Infect Dis 2003;3:359-371.

13. Dalhoff A. Immunomodulatory activities of fluoroquinolonesInfection 2005;33(suppl 2):55-70.

14. Kolios G, Manousou P, Bourikas L, et al. Ciprofloxacin inhibits cytokine-induced nitric oxide production in human colonic epithelium. Eur J Invest 2006;36:720-729.

15. Houdouin V, Bonacorsi S, Brahimi N, et al. An uropathogenicity island contributes to the pathogenicity of Escherichia coli strains that cause neonatal meningitis. Infect Immun 2002;70: 5865-5869.

16. Kim KS, Manocchio M, Bayer AS. Efficacy of cefotaxime and latamoxef for Escherichia coli bacteremia and meningitis in newborn rats. Chemotherapy 1984;30:262-269.

17. Klassen M, Edberg SC. Measurement of antibiotics in human body fluids: techniques and significance. In: Antibiotics in laboratory medicine. 4th ed. Baltimore: Williams \& Wilkins, 1996:230-295.

18. Trautmann M, Zick R, Rukavina $T$, et al. Antibiotic-induced release of endotoxin: in-vitro comparison of meropenem and other antibiotics. J Antimicrob Chemother 1998;41:163-169.

19. Baud O, Daire JL, Dalmaz Y, et al. Gestational hypoxia induces white matter damage in neonatal rats: a new model of periventricular leukomalacia. Brain Pathol 2004;14:1-10.

20. Fontaine $\mathrm{RH}$, Olivier $\mathrm{P}$, Massonneau $\mathrm{V}$, et al. Vulnerability of white matter towards antenatal hypoxia is linked to a species-dependent regulation of glutamate receptor subunits. Proc Natl Acad Sci USA 2008;105:16779-16784. 
21. Olivier P, Fontaine RH, Loron $\mathrm{G}$, et al. Melatonin promotes oligodendroglial maturation of injured white matter in neonatal rats. PLoS One 2009;4:e7128.

22. Chomczynski P, Sacchi N. Single-step method of RNA isolation by acid guanidinium thiocyanate-phenol-chloroform extraction. Anal Biochem 1987;162:156-159.

23. Spassky N, de Castro F, Le Bras B, et al. Directional guidance of oligodendroglial migration by class 3 semaphorins and netrin-1. J Neurosci 2002:22:5992-6004

24. Williams A, Piaton G, Aigrot MS, et al. Semaphorin $3 A$ and 3F: key players in myelin repair in multiple sclerosis? Brain 2007;130: 2554-2565

25. Schmitz T, Heep A, Groenendaal F, et al. Interleukin-1beta, interleukin-18, and interferon-gamma expression in the cerebrospinal fluid of premature infants with posthemorrhagic hydrocephalus-markers of white matter damage? Pediatr Res 2007; 61:722-726.

26. Whitelaw A, Thoresen M. Antenatal steroids and the developing brain. Arch Dis Child Fetal Neonatal Ed 2000;83:F154-F157.

27. Haynes RL, Baud O, Li J, et al. Oxidative and nitrative injury in periventricular leukomalacia: a review. Brain Pathol 2005;15: 225-233.

28. Kannan S, Saadani-Makki F, Muzik O, et al. Microglial activation in perinatal rabbit brain induced by intrauterine inflammation: detection with 11C-(R)-PK11195 and small-animal PET. J Nucl Med 2007;48:946-954.

29. Yuan TM, Yu HM, Gu WZ, Li JP. White matter damage and chemokine induction in developing rat brain after intrauterine infection. J Perinat Med 2005;33:415-422.

30. Debillon T, Gras-Leguen C, Leroy $\mathrm{S}$, et al. Patterns of cerebral inflammatory response in a rabbit model of intrauterine infectionmediated brain lesion. Brain Res Dev Brain Res 2003;145:39-48.

31. Dean JM, Farrag D, Zahkouk SA, et al. Cerebellar white matter injury following systemic endotoxemia in preterm fetal sheep. Neuroscience 2009:160:606-615.

32. Baud O, Greene AE, Li J, et al. Glutathione peroxidase-catalase cooperativity is required for resistance to hydrogen peroxide by mature rat oligodendrocytes. J Neurosci 2004;24:1531-1540.

33. Baud O, Haynes RF, Wang H, et al. Developmental up-regulation of MnSOD in rat oligodendrocytes confers protection against oxidative injury. Eur J Neurosci 2004;20:29-40.
34. Baud O, Li J, Zhang $Y$, et al. Nitric oxide-induced cell death in developing oligodendrocytes is associated with mitochondrial dysfunction and apoptosis-inducing factor translocation. Eur J Neurosci 2004;20:1713-1726.

35. Back SA, Han BH, Luo NL, et al. Selective vulnerability of late oligodendrocyte progenitors to hypoxia-ischemia. J Neurosci 2002; 22:455-463.

36. Feldhaus B, Dietzel ID, Heumann R, Berger R. Effects of interferon-gamma and tumor necrosis factor-alpha on survival and differentiation of oligodendrocyte progenitors. J Soc Gynecol Investig 2004;11:89-96.

37. Mann SA, Versmold B, Marx R, et al. Corticosteroids reverse cytokine-induced block of survival and differentiation of oligodendrocyte progenitor cells from rats. J Neuroinflammation 2008;5:39.

38. Foote AK, Blakemore WF. Inflammation stimulates remyelination in areas of chronic demyelination. Brain 2005;128:528-539.

39. Haynes RL, Folkerth RD, Trachtenberg FL, et al. Nitrosative stress and inducible nitric oxide synthase expression in periventricular leukomalacia. Acta Neuropathol 2009;118:391-399.

40. Riesbeck K, Forsgren A, Henriksson A, Bredberg A. Ciprofloxacin induces an immunomodulatory stress response in human $\mathrm{T}$ lymphocytes. Antimicrob Agents Chemother 1998;42:1923-1930.

41. Riesbeck K. Immunomodulating activity of quinolones: review. J Chemother 2002:14:3-12.

42. Gollapudi S, Kim CH, Roshanravan B, Gupta S. Ciprofloxacin inhibits activation of latent human immunodeficiency virus type 1 in chronically infected promonocytic U1 cells. AIDS Res Hum Retroviruses 1998;14:499-504.

43. Katsuno G, Takahashi HK, Iwagaki $H$, et al. The effect of ciprofloxacin on CD14 and toll-like receptor-4 expression on human monocytes. Shock 2006;25:247-253.

44. Ogino $H$, Fujii $M$, Ono $M$, et al. In vivo and in vitro effects of fluoroquinolones on lipopolysaccharide-induced pro-inflammatory cytokine production. J Infect Chemother 2009;15:168-173.

45. Gogos CA, Skoutelis A, Lekkou A, et al. Comparative effects of ciprofloxacin and ceftazidime on cytokine production in patients with severe sepsis caused by gram-negative bacteria. Antimicrob Agents Chemother 2004;48:2793-2798.

46. Holzheimer RG. The significance of endotoxin release in experimental and clinical sepsis in surgical patients-evidence for antibiotic-induced endotoxin release? Infection 1998;26:77-84. 